\title{
Superradiant light scattering from a moving Bose-Einstein condensate
}

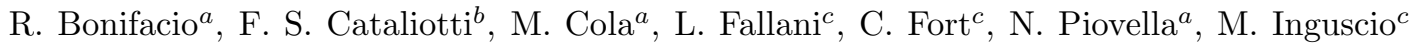 \\ INFM and European Laboratory for Non-Linear Spectroscopy (LENS) \\ via N. Carrara 1, I-50019 Sesto F.no (FI), Italy \\ a) Dipartimento di Fisica, Università degli Studi di Milano, Via Celoria 16, I-20133 Milano, Italy \\ b)Dipartimento di Fisica, Università di Catania, via S. Sofia 64, I-95124 Catania, Italy \\ c)Dipartimento di Fisica, Università di Firenze, via G. Sansone 1, I-50019 Sesto F.no (FI), Italy
}

(Dated: September 12, 2018)

\begin{abstract}
We investigate the interaction of a moving BEC with a far detuned laser beam. Superradiant Rayleigh scattering arises from the spontaneous formation of a matter-wave grating due to the interference of two wavepackets with different momenta. The system is described by the CARLBEC model which is a generalization of the Gross-Pitaevskii model to include the self-consistent evolution of the scattered field. The experiment gives evidence of a damping of the matter-wave grating which depends on the initial velocity of the condensate. We describe this damping in terms of a phase-diffusion decoherence process, in good agreement with the experimental results.
\end{abstract}

The experimental realization of Bose-Einstein condensates (BECs) with alkali trapped atoms has opened the possibility of investigating several fundamental aspects of quantum mechanics in macroscopic, i.e. many particle systems [1]. In superradiant Rayleigh scattering the coherent nature of the condensate leads to strong correlations between successive scattering events, as shown in the pioneering work of Ketterle and coworkers [2]. This process was then the basis for the first demonstration of phase-coherent matter wave amplification [3]. The effect studied by Ketterle is an example of a spontaneous formation of a regular density grating in an atomic system, arising from a collective instability as in the Collective Atomic Recoil Laser (CARL) [4]. In the absence of thermal broadening (as it happens in a BEC), CARL appears as a promising source of macroscopically entangled or number-squeezed atom-atom and/or atomphoton systems [5, 6, 7]. However, in a real BEC several effects due, for instance, to spontaneous emission, inhomogeneous broadening and collisions, may seriously inhibit the CARL process and destroy the coherence in the matter wave field [8]. The control of decoherence in the photon-BEC interaction would be a significant step toward the achievement of macroscopic entanglement of coherent matter waves.

In this paper we investigate both theoretically and experimentally the influence of the initial velocity of the condensate on superradiant Rayleigh scattering. In the experiment we produce an elongated BEC of rubidium atoms and expose it to a single off-resonant laser pulse directed along the condensate symmetry axis. The laser is far detuned from any atomic resonance and the only scattering mechanism present is Rayleigh scattering [2]. In an elongated condensate a preferential direction for the scattered photons emerges, causing superradiant Rayleigh scattering. In this regime the atoms, initially scattered randomly, interfere with the atoms in the original momentum state creating a matter-wave grating with the right periodicity to further scatter the laser photons in the same mode. Both the matter-wave grating and the scattered light are then coherently amplified. In our ge- ometry photons are back-scattered with $\vec{k}_{s} \approx-\vec{k}$, where $\vec{k}$ is the wave-vector of the laser photon, and the atoms move away from the original condensate with a relative momentum $2 \hbar k$ in the direction of the laser beam. The efficiency of the process is limited by the decoherence between the original and the recoiled atomic wavepackets causing the damping of the matter-wave grating. We identify two different mechanisms for decoherence, one resulting from Doppler and mean field broadening of the matter wave field [2, 9] and the other due to phase diffusion. The latter mechanism, dependent on the energy separation between the initial and final states of the system 10, 11], can be controlled by initially setting the condensate into motion. In particular, we observe that phase diffusion decoherence vanishes when the initial condensate momentum is such that after the interaction with the laser beam the scattered atomic wavepacket has the same kinetic energy of the original condensate in the laboratory frame.

The experiment is performed with a cigar-shaped condensate of ${ }^{87} \mathrm{Rb}$ produced in a Ioffe-Pritchard magnetic trap by means of RF-induced evaporative cooling. The axial and radial frequencies of the trap are $\omega_{z} / 2 \pi=$ $8.70(7) \mathrm{Hz}$ and $\omega_{r} / 2 \pi=90.1(4) \mathrm{Hz}$ respectively, with the $z$-axis oriented horizontally. We tune the atomic velocity after the end of the evaporative ramp by inducing a collective dipole motion of the condensate along the $z$-axis. The dipole oscillation is excited by non-adiabatically displacing the center of the magnetic trap. When the condensate has reached the maximum velocity in the magnetic potential, we suddenly switch off the trap and let the cloud expand with a horizontal velocity proportional to the displacement of the trap (see Fig [1). We apply a square pulse of light along the $z$-axis, $2 \mathrm{~ms}$ after the release of the condensate, when the magnetic field of the trap is completely switched off, and the atomic cloud has still an elongated shape. After $2 \mathrm{~ms}$ of expansion the radial and axial sizes of our condensates are typically 10 and $70 \mu \mathrm{m}$, respectively. The pulse length is controlled with an acousto-optic modulator. The light comes from a diode laser red-detuned $13 \mathrm{GHz}$ away from 
(A)

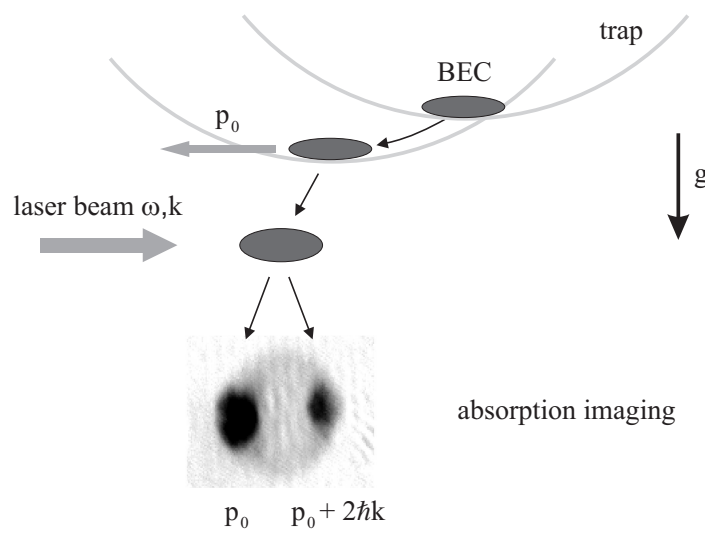

FIG. 1: Schematics of the experimental procedure. The condensate is set in motion by a sudden displacement of the magnetic trap center (A). When the condensate reaches the desired momentum $\mathrm{p}_{0}$ we switch off the magnetic trap and flash the atoms with a far off resonance laser pulse directed along the condensate symmetry axis (B). After an expansion time allowing a complete separation of the momentum components (28 ms) we take an absorption image of the atoms (C).

the rubidium D2 line at $\lambda=780 \mathrm{~nm}$ and has an intensity of $1.35 \mathrm{~W} / \mathrm{cm}^{2}$ corresponding to a Rayleigh scattering rate of roughly $5 \times 10^{2} \mathrm{~s}^{-1}$. The linearly polarized laser beam is collimated and aligned along the $z$-axis of the condensate. In this geometry the superradiant light is backscattered and the self-amplified matter-wave propagates in the same direction of the incident light. In order to minimize spurious reflections we have aligned the laser beam at a nonzero angle with respect to the normal to the vacuum cell windows. After an expansion of $28 \mathrm{~ms}$, when the two momentum components are spatially separated, we take an absorption image of the cloud along the horizontal radial direction.

In Fig. 1C we show a typical absorption image in which the left peak is the condensate in its original momentum state $p_{0}$ and the right peak is formed by atoms recoiling after the superradiant scattering at $p_{0}+2 \hbar k$. The spherical halo centered between the two density peaks is due to non-enhanced spontaneous processes, i.e. random isotropic emission following the absorption of one laser photon. From a 2D-fit of the pictures assuming a Thomas-Fermi density distribution we extract the num- ber of atoms in both the original and the recoiled peaks.

To understand the observed behavior we analyze in detail the self-consistent interaction between a coherent electromagnetic wave of amplitude $a$ and a coherent matter wave field $\Psi$. The evolution of the system is described by the following 1-D CARL-BEC model, i.e. a Gross-Pitaevskii model generalized to include the selfconsistent evolution of the scattered radiation amplitude:

$$
\begin{aligned}
i \hbar \frac{\partial \Psi}{\partial t} & =-\frac{\hbar^{2}}{2 m} \frac{\partial^{2} \Psi}{\partial z^{2}}+\beta N|\Psi|^{2} \Psi \\
& +i \hbar g\left\{a^{*} e^{i(2 k z-\delta t)}-\text { c.c. }\right\} \Psi \\
\frac{d a}{d t} & =g N \int d z|\Psi|^{2} e^{i(2 k z-\delta t)}-\kappa a .
\end{aligned}
$$

In Eqs.(11) and (2), $a(t)=\left(\epsilon_{0} V / 2 \hbar \omega_{s}\right)^{1 / 2} E_{s}(t)$ is the dimensionless electric field amplitude of the scattered beam with frequency $\omega_{s}, g=(\Omega / 2 \Delta)\left(\omega d^{2} / 2 \hbar \epsilon_{0} V\right)^{1 / 2}$ is the coupling constant, $\Omega$ is the Rabi frequency of the laser beam with a frequency $\omega$ detuned from the atomic resonance frequency $\omega_{0}$ by $\Delta=\omega-\omega_{0}, d=\hat{\epsilon} \cdot \vec{d}$ is the electric dipole moment of the atom along the polarization direction $\vec{\epsilon}$ of the laser, $V$ is the volume of the condensate, $N$ is the total number of atoms in the condensate and $\delta=\omega-\omega_{s}$. The matter wave field is normalized such that $\int d z|\Psi|^{2}=1$. The nonlinear term in Eq. (11) can be neglected since the experiment has been performed after expansion. The last term in right-hand side of Eq.(1) represents the self-consistent optical wave grating, whose amplitude depends on time according to Eq.(2). The first term in the right-hand side of Eq.(2) represents the selfconsistent matter-wave grating. Eq.(2) has been written in the "mean-field" limit, which models the propagation effects with a damping term where $\kappa \approx c / 2 L$ and $L$ is the condensate length [12].

If the condensate is much longer than the radiation wavelength and approximately homogeneous, then periodic boundary conditions can be assumed and the wavefunction can be written as $\Psi(z, t)=$ $\sum_{n} c_{n}(t) u_{n}(z) e^{-i n \delta t}$, where $u_{n}(z)=(2 / \lambda)^{1 / 2} \exp (2 i n k z)$ are the momentum eigenstates with eigenvalues $p_{z}=$ $n(2 \hbar k)$. Introducing the density matrix $\rho_{m, n}=c_{m} c_{n}^{*}$ and $\omega_{n}=4 \omega_{R} n^{2}-\delta n$, where $\omega_{R}=\hbar k^{2} / 2 m$ is the recoil frequency, we obtain from Eqs.(11) and (2):

$$
\begin{aligned}
\frac{d \rho_{m, n}}{d t} & =-i\left(\omega_{m}-\omega_{n}\right) \rho_{m, n}+g\left\{a\left(\rho_{m, n-1}-\rho_{m+1, n}\right)+a^{*}\left(\rho_{m-1, n}-\rho_{m, n+1}\right)\right\}-\frac{\tau}{2}\left(\omega_{m}-\omega_{n}\right)^{2} \rho_{m, n} \\
\frac{d a}{d t} & =g N \sum_{n} \rho_{n, n+1}-\kappa a .
\end{aligned}
$$


by a constant $\tau$. This term, fundamental to describe our experimental results, arises from a $\delta$-correlated gaussian noise on the eigenenergies of the system and causes the decay of the off-diagonal matrix elements, so that the density matrix becomes diagonal in the basis of the recoil momentum states. Eq.(3) may be written as a master equation [14] for the density operator $\hat{\rho}=$ $\sum_{m, n} \rho_{m, n}|m\rangle\langle n|:$

$$
\frac{d \hat{\rho}}{d t}=-\frac{i}{\hbar}[\hat{H}, \hat{\rho}]-\frac{\tau}{2}\left[\hat{H}_{0},\left[\hat{H}_{0}, \hat{\rho}\right]\right],
$$

where $\hat{H}=\hat{H}_{0}+\hat{V}, \hat{H}_{0}=4 \hbar \omega_{R} \hat{p}^{2}-\hbar \delta \hat{p}, \hat{V}=$ $i \hbar g\left(a^{*} e^{2 i k z}-h . c.\right)$ and $\hat{p}=p_{z} / 2 \hbar k$ is the normalized momentum operator with eigenstates $|n\rangle$ and eigenvalues $n$. The phase destroying term with the double commutator of the Lindblad form in the right-hand side of Eq.(5) generates the damping term added in Eq.(3). It has appeared in many models of decoherence and induces diffusion in variables that do not commute with the Hamiltonian, preserving the number of atoms in the condensate. In this term we have neglected the interaction $\hat{V}$ in the weak-coupling limit $g^{2} N / \kappa \ll \omega_{R}$.

In our experimental conditions the superradiant Rayleigh scattering involves only neighboring momentum states, i.e. transitions from the initial momentum state $p_{0}=n(2 \hbar k)$ to the final momentum state $(n+1) 2 \hbar k$. In this limit, our system is equivalent to a two-level system and Eqs.(33) and (4) reduce to a set of equations formally equivalent to the well-known Maxwell-Bloch system [9, 15]:

$$
\begin{aligned}
& \frac{d S}{d t}=g A W-\gamma_{n} S \\
& \frac{d W}{d t}=-2 g\left(A S^{*}+h . c .\right) \\
& \frac{d A}{d t}=g N S-\left(\kappa-i \Delta_{n}\right) A,
\end{aligned}
$$

where $S=\rho_{n, n+1} e^{i \Delta_{n} t}, A=a e^{i \Delta_{n} t}, W=P_{n}-P_{n+1}$ is the population fraction difference between the two states (where $P_{n}=\rho_{n, n}$ and $P_{n}+P_{n+1}=1$ ), $\Delta_{n}=\omega-\omega_{s}-$ $4 \omega_{R}(2 n+1)$ and the decoherence rate $\gamma_{n}$ is given by:

$$
\gamma_{n}=\gamma_{0}+\frac{\tau}{2} \Delta_{n}^{2}=\gamma_{0}+\frac{\tau}{2}\left[\omega-\omega_{s}-4 \omega_{R}\left(\frac{p_{0}}{\hbar k}+1\right)\right]^{2} .
$$

To the decoherence rate $\gamma_{n}$ we have added an extra term $\gamma_{0}$ taking into account other coherence decay mechanisms, as for instance Doppler and inhomogeneous broadenings of the two-photon Bragg resonance [2, 9]. We note that in Eq. (8), $S$ represents half of the amplitude of the matter-wave grating. In fact, if $\Psi \approx c_{n} u_{n}(z)+c_{n+1} u_{n+1}(z)$, the longitudinal density is $|\Psi|^{2} \approx(2 / \lambda)\left\{1+2 \operatorname{Re}\left[S^{*} \exp \left(2 i k z-i \Delta_{n} t\right)\right]\right\}$, which describes a matter wave grating with a periodicity of half the laser wavelength. The main result is that the second term of Eq.(9), arising from a phase diffusion decoherence mechanism, depends on the frequency detuning between

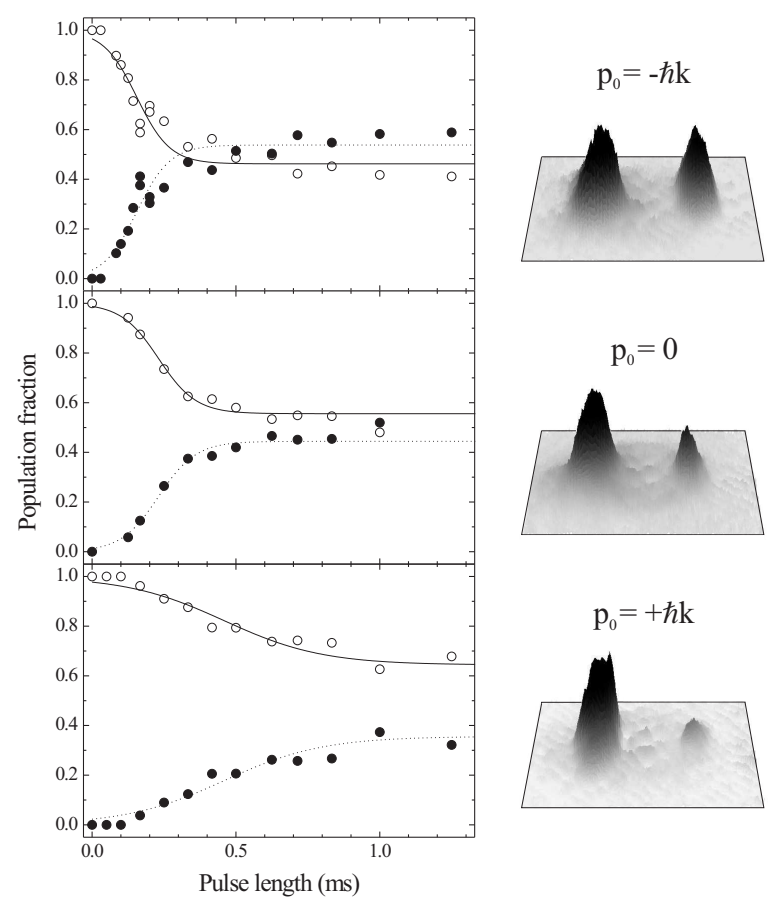

FIG. 2: Left) Time evolution of population in the original condensate (empty circles) and in the recoiled wavepacket (filled circles) for different pulse durations. The solid line is a fit with the hyperbolic tangent (10) predicted by the theoretical model, the dotted line is just one minus the fit curve. The momentum of the original condensate is set to $-\hbar k$ (top), 0 (center) and $+\hbar k$ (bottom). Right) Plot of the atomic density profile after interaction with a $250 \mu \mathrm{s}$ pulse for the three cases of original momentum as on the left. The laser detuning and intensity are $13 \mathrm{GHz}$ and $1.35 \mathrm{~W} / \mathrm{cm}^{2}$ respectively.

the incident and scattered radiation beams and on the initial momentum of the condensate, $p_{0}=n(2 \hbar k)$. We observe that the velocity-dependent term of the decoherence rate is invariant under Galilean transformation. In fact, in a frame moving with respect to the laboratory frame, the shift of $p_{0}$ compensates the Doppler shift of the frequency difference $\omega-\omega_{s}$.

Our experimental conditions match those for the superfluorescent regime [13], in which the field loss rate $\kappa$ is much larger than the coupling rate $g \sqrt{N}$. In this regime, for $t \gg \kappa^{-1}$, we can perform the adiabatic elimination $A \simeq g N S /\left(\kappa-i \Delta_{n}\right)$. The analytical solution for the fraction of atoms with initial momentum $p_{0}=n(2 \hbar k)$ is

$$
\begin{aligned}
P_{n} & =1-\frac{1}{2}\left(1-\frac{2 \gamma_{n}}{G}\right) \times \\
& \times\left\{1+\tanh \left[\left(G-2 \gamma_{n}\right)\left(t-t_{0}\right) / 2\right]\right\},
\end{aligned}
$$

where $G=2 g^{2} N \kappa /\left(\kappa^{2}+\Delta_{n}^{2}\right)$ is the superradiant gain and $t_{0}$ is a delay time. In our experiment $\kappa \gg \Delta_{n}$, so that $G \approx 2 g^{2} N / \kappa$ hence independent from the atomic veloc- 


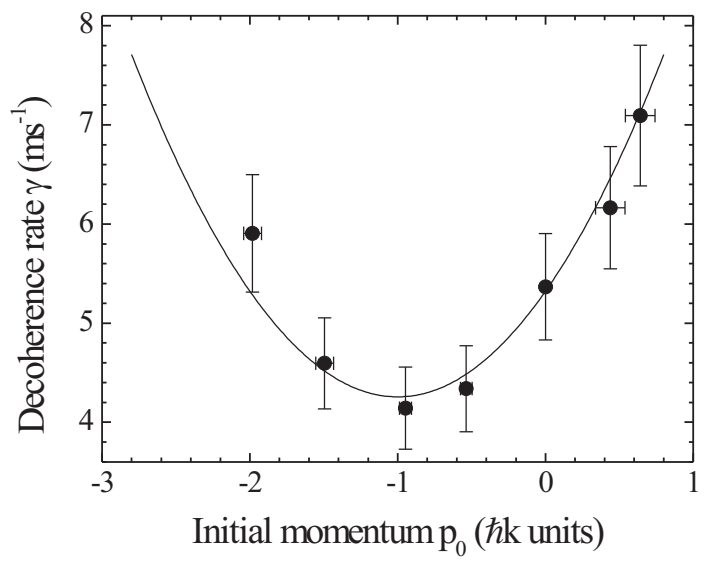

FIG. 3: Decoherence rate as a function of the initial momentum of the condensate. The solid line is a fit of the experimental data with a parabola centered in $\mathrm{p}_{0}=-\hbar k$, as expected from the theoretical model.

ity. Eq. (10) assumes the threshold condition $G>2 \gamma_{n}$ i.e. the gain must be larger than the decoherence rate. Fig 2 reports the population fractions of the initial wavepacket, $P_{n}$, and of the scattered wavepacket, $P_{n+1}$, as functions of the laser pulse duration for three different initial momenta $p_{0}$. ¿From the fits we extract the values of $G$ and $\gamma_{n}$ for different $p_{0}$. The measured value of $G=19(3)$ $\mathrm{ms}^{-1}$ does not appreciably depend on $p_{0}$. On the contrary we observe a strong dependence of the decoherence rate $\gamma_{n}$ on the initial momentum $p_{0}$. In Fig 3 we plot the experimental points for the decoherence rate $\gamma_{n}$ as a function of the initial momentum of the atoms. The data show a parabolic behavior in good agreement with the prediction of Eq. (9) if one assumes $\omega=\omega_{s}$ in the laboratory frame, with fit values $\gamma_{0}=4.2(2) \mathrm{ms}^{-1}$ and $\tau=2.4(2) \cdot 10^{-7} \mathrm{~s}$. The theoretical calculation of the linewidth of the Bragg resonance 16] for our experimental parameters predicts a value $\gamma_{0} \approx 3 \mathrm{~ms}^{-1}$ close to the value obtained from the fit in Fig 3 Notice that the decoherence rate is minimized for $p_{0}=-\hbar k$. Indeed, if the initial momentum is $-\hbar k$, after scattering the atoms have the same kinetic energy and, with the above assumption for the scattered light frequency $\omega_{s}$, the phase-diffusion decoherence term in Eq.(9) is zero. This identifies a subspace which is decoherence free with respect to the phase destroying process [17].

In our experimental apparatus it is very difficult to completely exclude the presence of pump light diffused by the vacuum cell windows. We have measured the diffused light present in our chamber in the direction opposite to the pump beam to be $1.0(3) \times 10^{-5}$ of the pump beam intensity. This amount of light is of the same order of magnitude of the equivalent input noise of the superradiant process [18]. This justifies the assumption that $\omega_{s}=\omega$ used to fit the experimental data of Fig. 3 We remark that this back diffused light can not explain our results in terms of Bragg scattering since the width of the Bragg resonance is one order of magnitude smaller than the range of momenta explored in our experiment and shown in Fig. 3] Furthermore the hyperbolic tangent dependence of the atomic population in Fig. 2 can only be explained by the self consistent amplification of the matter wave grating and of the backscattered light as described in Eqs.(11) and (2).

In conclusion, we have studied the superradiant light scattering from a moving Bose-Einstein condensate. The efficiency of the overall process is fundamentally limited by the decoherence between the two atomic momentum states. With the assumption for the scattered light frequency $\omega_{s}=\omega$ in the laboratory frame, Eq. (9) predicts the parabolic behavior of the decoherence rate as a function of the initial momentum, in agreement with the experimental results as shown in Fig 3 The fully quantized version of the CARL-BEC model offers the possibility of investigating the realisation of macroscopic atom-atom or atom-photon entanglement [5, [6]. The control of decoherence obtained in this work represents a significant step in this direction.

This work has been supported by the EU, INFM and MIUR. We thank B. Englert and S. Olivares for useful discussions.
[1] See for example Bose-Einstein Condensation in Atomic Gases, M. Inguscio, C. E. Wieman, S. Stringari, Eds. (IOS Press Amsterdam, Oxford, Tokyo, Washington, 1999).

[2] S. Inouye, A. P. Chikkatur, D. M. Stamper-Kurn, J.Stenger, D. E. Pritchard and W. Ketterle, Science 285, 571 (1999).

[3] M. Kozuma, Y. Suzuki, Y. Torii, T. Sugiura, T. Kuga, E. W. Hagley and L. Deng, Science 2862309 (1999); S. Inouye, T. Pfau, S. Gupta, A. P. Chikkatur, A. Gorlitz, D. E. Pritchard and W. Ketterle, Nature 402641 (1999).

[4] R. Bonifacio and L. De Salvo, Nucl. Instrum. Methods Phys Res. A 341, 360 (1994); R. Bonifacio, L. De Salvo, L. M. Narducci and E. J. D'Angelo, Phys. Rev. A 50,
1716 (1994).

[5] M.G. Moore, O. Zobay and P. Meystre, Phys. Rev. A 60, 1491 (1999).

[6] N. Piovella, M. Cola and R. Bonifacio, Phys. Rev. A 67, 013817 (2003).

[7] J. M. Vogels, K. Xu, and W. Ketterle, Phys. Rev. Lett., 89020401 (2002).

[8] T. Gasenzer, D. C. Roberts, and K. Burnett, Phys. Rev. A 65 021605R (2002).

[9] S. Inouye, R.F. Low, S. Gupta, T.Pfau, A.Gorlitz, T.L. Gustavson, D. E. Pritchard and W. Ketterle, Phys. Rev. Lett. 85, 4225 (2000).

[10] W. H. Zurek, Phys. Rev. D 24, 1516 (1981).

[11] R. Bonifacio, Il Nuovo Cimento B 114, 473 (1999). 
[12] R. Bonifacio, P. Schwendimann and F. Haake, Phys. Rev. A 4, 302 (1971); 4, 854 (1971).

[13] R. Bonifacio and L.A. Lugiato, Phys. Rev. A 11, 1507 (1975).

[14] D.F. Walls and G.J. Milburn, Phys. Rev. A 31, 2403 (1985).

[15] N. Piovella, M. Gatelli and R. Bonifacio, Optics Comm. 194, 167 (2001).

[16] J. Stenger, S. Inouye, A.P. Chikkatur, D.M. Stamper-
Kurn, D.E. Pritchard and W. Ketterle, Phys. Rev. Lett. 82, 4569 (1999).

[17] See for example Experimental Quantum Computation and Information, F. De Martini and C. Monroe, Eds. (IOS Press Amsterdam, Oxford, Tokyo, Washington, 2003).

[18] M. Cola, PhD Thesis (2003). 